\title{
Products and ratios of characteristic polynomials of random Hermitian matrices
}

\author{
Jinho Baik ${ }^{\mathrm{a})}$ \\ Department of Mathematics, Princeton University, Princeton, New Jersey 08544 \\ and Department of Mathematics, University of Michigan, Ann Arbor, Michigan 48109 \\ Percy Deift $\left.{ }^{\mathrm{b}}\right)$ \\ Courant Institute of Mathematical Sciences, New York University, \\ New York, New York 10012 \\ and School of Mathematics, Institute for Advanced Study, \\ Princeton, New Jersey 08540 \\ Eugene Strahov ${ }^{\mathrm{c}}$ \\ Department of Mathematical Sciences, Brunel University, \\ Uxbridge, UB8 3PH, United Kingdom
}

(Received 7 April 2003; accepted 9 April 2003)

We present new and streamlined proofs of various formulas for products and ratios of characteristic polynomials of random Hermitian matrices that have appeared recently in the literature. (C) 2003 American Institute of Physics.

[DOI: 10.1063/1.1587875]

\section{INTRODUCTION}

In random matrix theory, unitary ensembles of $N \times N$ matrices $\{H\}$ play a central role. ${ }^{15}$ Such ensembles are described by a measure $d \alpha$ with finite moments $\int_{\mathrm{R}}|x|^{k} d \alpha(x)<\infty, k=0,1,2, \ldots$, and the associated distribution function for the eigenvalues $\left\{x_{i}=x_{i}(H)\right\}$ of matrices $H$ in the ensembles has the form

$$
d \mathrm{P}_{\alpha, N}(x)=\frac{1}{Z_{N}} \Delta(x)^{2} d \alpha(x),
$$

where $d \alpha(x)=\Pi_{i=1}^{N} d \alpha\left(x_{i}\right), \Delta(x)=\Pi_{N \geqslant i>j \geqslant 1}\left(x_{i}-x_{j}\right)$ is the Vandermonde determinant for the $x_{i}$ 's, and $Z_{N}=\int \cdots \int \Delta(x)^{2} d \alpha(x)$ is the normalization constant. The special case $d \alpha(x)$ $=e^{-x^{2}} d x$ is known as the Gaussian unitary ensemble (GUE). For symmetric functions $f(x)$ $=f\left(x_{1}, \ldots, x_{N}\right)$ of the $x_{i}$ 's,

$$
\langle f\rangle_{\alpha} \equiv \frac{1}{Z_{N}} \int \cdots \int f(x) \Delta(x)^{2} d \alpha(x)
$$

denotes the average of $f$ with respect to $d \mathrm{P}_{\alpha, N}$.

Recently there has been considerable interest in the averages of products and ratios of the characteristic polynomials $D_{N}[\mu, H]=\Pi_{i=1}^{N}\left(\mu-x_{i}(H)\right)$ of random matrices with respect to various ensembles. Such averages are used, in particular, in making predictions about the moments of the Riemann-zeta function [see Refs. 12-14 (circular ensembles) and 3 (unitary ensembles)]. Many other uses are described, for example, in Refs. 1, 12, and 17.

By (1.2), for unitary ensembles, such averages have the form

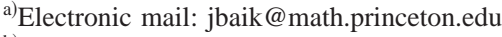

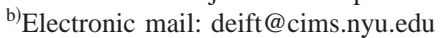

${ }^{c)}$ Electronic mail: eugene.strahov@brunel.ac.uk
} 


$$
\left|\frac{\prod_{j=1}^{K} D_{N}\left[\mu_{j}, H\right]}{\prod_{j=1}^{M} D_{N}\left[\epsilon_{j}, H\right]}\right|_{\alpha}=\frac{1}{Z_{N}} \int \cdots \int \frac{\prod_{j=1}^{K} \prod_{i=1}^{N}\left(\mu_{j}-x_{i}\right)}{\prod_{j=1}^{M} \prod_{j=1}^{N}\left(\epsilon_{j}-x_{i}\right)} \Delta(x)^{2} d \alpha(x) .
$$

In this article we consider certain explicit determinantal formulas for (1.3) - see (2.6), (2.24), (2.36), (3.3), and (3.12) below. Formula (2.6) is due to Brezin and Hikami ${ }^{3}$ (see also Ref. 16, and when all the $\mu_{j}$ 's are equal, see Ref. 10), whereas (2.24), (2.36), (3.3), and (3.12) are due to Fyodorov and Strahov. ${ }^{17,11}$ References 17 and 11 also contain a discussion of the history of these formulas. The formulas (3.3) and (3.12) are particularly useful in proving universality results for the ratios (1.3) in the Dyson limit as $N \rightarrow \infty$ (see Ref. 17). For a discussion of other universality results, particularly the work of Brezin-Hikami and Fyodorov in special cases, we again refer the reader to Ref. 17. The asymptotic analysis in Ref. 17 is based on the reformulation of the orthogonal polynomial problem as a Riemann-Hilbert problem by Fokas, Its, and Kitaev. ${ }^{9}$ The RiemannHilbert problem is then analyzed asymptotically using the noncommutative steepest-descent method introduced by Deift and Zhou, ${ }^{5}$ and further developed with Venakides in Ref. 6 to allow for fully nonlinear oscillations, and in Refs. 7 and 8.

Our goal in this article is to give new, streamlined proofs of (2.6)-(3.12), using only the properties of orthogonal polynomials and a minimum of combinatorics. Along the way we will also need an integral version of the classical Binet-Cauchy-formula due to C. Andréief dating back to 1883 (see Lemma 2.1 below).

Let $\pi_{j}(z)=x^{j}+\cdots$ denote the $j$ th monic orthogonal polynomial with respect to the measure $d \alpha$,

$$
\int_{\mathrm{R}} \pi_{j}(x) \pi_{k}(x) d \alpha(x)=c_{j} c_{k} \delta_{j k} ; \quad j, k \geqslant 0,
$$

where the norming constants $c_{j}$ 's are positive. The key observation in our approach is that for $K=1$ and $M=0$ in (1.3),

$$
\left\langle D_{N}[\mu, H]\right\rangle_{\alpha}=\pi_{N}(\mu)
$$

(see Ref. 18). In our words, the orthogonal polynomial $\pi_{N}(\mu)$ with respect to $d \alpha$ is also precisely the average polynomial $\Pi_{i=1}^{N}\left(\mu-x_{i}\right)$ with respect to $d \mathrm{P}_{\alpha, N}$. Formula (1.5) appears already in the work of Heine in the 1880s (see Ref. 18). Set

$$
d \alpha^{[\ell, m]}(t) \equiv \frac{\prod_{j=1}^{\ell}\left(\mu_{j}-t\right)}{\prod_{j=1}^{m}\left(\epsilon_{j}-t\right)} d \alpha(t), \quad \ell, m \geqslant 0,
$$

$\left[d \alpha^{[0,0]}(t) \equiv d \alpha(t)\right]$, and let $\pi_{j}^{[\ell, m]}(t)$ denote the $j$ th monic orthogonal polynomial with respect to $d \alpha^{[\ell, m]}$. With this notation we see immediately from (1.3) and (1.5) that $\left\langle\mathrm{Q}_{j=1}^{K} D_{N}\left[\mu_{j}, H\right] / \mathrm{Q}_{j=1}^{M} D_{N}\left[\epsilon_{j}, H\right]\right\rangle_{\alpha}$ is proportional to $\pi_{N}^{[K-1, M]}\left(\mu_{K}\right)$. Using a classical determinantal formula of Christoffel (see Ref. 18) for $\pi_{N}^{[\ell, 0]}(\mu)$ and a more recent formula of Uvarov ${ }^{19}$ for $\pi_{N}^{[0, m]}(\mu)$, we are then led (see Sec. II) to (2.6), (2.24), and (2.36) in a rather straightforward way. Formula (3.3) appears to have a different character from (2.6), (2.24), and (2.36), and relies on Lemma 2.1 mentioned above, which computes the integral of the product of two determinants: formula (3.12) follows (see Sec. III) by combining (3.3) with (2.6) and (2.36). In Ref. 17 the authors present a variety of additional formulas for $\left\langle\mathrm{Q}_{j=1}^{K} D_{N}\left[\mu_{j}, H\right] / \mathrm{Q}_{j=1}^{M} D_{N}\left[\epsilon_{j}, H\right]\right\rangle_{\alpha}$ for cases of $K$ and $M$ not covered by (2.6)-(3.12): we leave it to the interested reader to verify that the method of this article can also be used to derive these formulas in a straightforward manner.

Remark 1.1: As is well known (see, e.g., Ref. 18), each measure $d \alpha$ gives rise to a tridiagonal operator 


$$
J=J(d \alpha)=\left(\begin{array}{cccc}
a_{1} & b_{1} & 0 & \\
b_{1} & a_{2} & b_{2} & \\
0 & b_{2} & a_{3} & \ddots \\
& & \ddots & \ddots
\end{array}\right), \quad b_{i}>0
$$

with generalized eigenfunctions given by the orthonormal polynomials

$$
p_{j}(x)=c_{j}^{-1} \pi_{j}(x), \quad j=0,1, \ldots,
$$

i.e.,

$$
b_{j-1} p_{j-1}(x)+a_{j} p_{j}(x)+b_{j} p_{j+1}(x)=x p_{j}(x), \quad j \geqslant 1,
$$

where $b_{0} \equiv 0$. Conversely, modulo certain essential self-adjointness issues, $d \alpha$ is the spectral measure for $J$ in the cyclic subspace generated by $J$ and the vector $e_{1}(1,0,0, \ldots)^{T}$ (see, e.g., Ref. 4). It follows that the transformation of measures

$$
d \alpha \rightarrow d \alpha^{[\ell, m]}
$$

leads to the transformation of operators

$$
J(d \alpha) \rightarrow J\left(d \alpha^{[\ell, m]}\right) .
$$

For appropriate choices of $\mu_{1}, \ldots, \mu_{m}$ and $\epsilon_{1}, \ldots, \epsilon_{\ell}$, such transformations correspond to removing $m$ points from the spectrum of $J(d \alpha)$ and inserting $\ell$ points: in the spectral theory literature, such transformations are known as Darboux transformations. The formulas in this article clearly provide formulas for the generalized eigenfunctions $p_{j}^{[\ell, m]}(x)$ of the Darboux-transformed operator $J\left(d \alpha^{[\ell, m]}\right)$, as well as the matrix entries, $a_{j}^{[\ell, m]}$ and $b_{j}^{[\ell, m]}$, in terms of the corresponding objects for $J(d \alpha)$. Again we leave the details to the reader. Here the elementary formulas

$$
b_{n}^{2}(d \alpha)=\frac{n+1}{n+2} \frac{Z_{n}(d \alpha) Z_{n+2}(d \alpha)}{\left(Z_{n+1}(d \alpha)\right)^{2}}, \quad a_{n}(d \alpha)=\left.\frac{d}{d t}\right|_{t=0} \log \frac{Z_{n}\left(d \alpha_{t}\right)}{Z_{n+1}\left(d \alpha_{t}\right)},
$$

where $d \alpha_{t}(x)=e^{t x} d \alpha(x)$, are useful.

Technical Remark 1.2: Formulas (2.6)-(3.12) clearly do not make sense for all values of the parameters. In all the calculations that follow, we will assume that $d \alpha$ has compact support, support $(d \alpha)=[-Q, Q]$, say, and that the $\mu_{i}$ 's and $\epsilon_{j}$ 's are distinct real numbers greater than $Q$ : under these assumptions, $d \alpha^{[\ell, m]}(t)$ becomes, in particular, a bona fide measure, etc. By analytic continuation one sees that the formulas remain true for complex values of $\left\{\mu_{i}\right\}$ and $\left\{\epsilon_{j}\right\}$, as long as they remain distinct. Furthermore, if the $\mu_{j}$ 's and $\epsilon_{j}$ 's are distinct, and $\operatorname{Im}\left(\epsilon_{j}\right) \neq 0$ for all $j$, then we can let $Q \rightarrow \infty$ and so the formulas are true for measures $d \alpha$ with unbounded support. Finally, we can, for example, let $\mu_{j} \rightarrow \mu_{k}$ for some $j \neq k$, which leads to formulas involving derivatives of the $\pi_{j}$ 's, etc.

\section{FORMULAS OF CHRISTOFFEL-UVAROV TYPE}

We use the notations $d \alpha, \pi_{j}, d \alpha^{[\ell, m]}, \pi_{j}^{[\ell, m]}, \ldots$ of Sec. I. In addition, in all the calculations that follow we assume that $d \alpha,\left\{\mu_{j}\right\},\left\{\epsilon_{k}\right\}$ satisfy the conditions described in Technical Remark 1.2 above: the natural analytical continuation of the formulas obtained to complex values of the parameters, and the limit $Q \rightarrow \infty$, is left to the reader.

The following result of Christoffel (see Ref. 18) plays a basic role in what follows.

Lemma 2.1: Consider the measure $d \alpha^{[\ell, 0]}(t)=\prod_{j=1}^{\ell}\left(\mu_{j}-t\right) d \alpha(t)$, where $\ell=1,2, \ldots$. Then the $n$th monic orthogonal polynomial $\pi_{n}^{[\ell, 0]}(t)$ associated with the new measure $d \alpha^{[\ell, 0]}(t)$ can be expressed as follows: 


$$
\pi_{n}^{[\ell, 0]}(t)=\frac{1}{\left(t-\mu_{1}\right) \cdots\left(t-\mu_{\ell}\right)} \frac{\left|\begin{array}{ccc}
\pi_{n}\left(\mu_{1}\right) & \cdots & \pi_{n+\ell}\left(\mu_{1}\right) \\
\vdots & & \\
\pi_{n}\left(\mu_{\ell}\right) & \cdots & \pi_{n+\ell}\left(\mu_{\ell}\right) \\
\pi_{n}(t) & \cdots & \pi_{n+\ell}(t)
\end{array}\right|}{\left|\begin{array}{ccc}
\pi_{n}\left(\mu_{1}\right) & \cdots & \pi_{n+\ell-1}\left(\mu_{1}\right) \\
\vdots & & \\
\pi_{n}\left(\mu_{\ell}\right) & \cdots & \pi_{n+\ell-1}\left(\mu_{\ell}\right)
\end{array}\right|} .
$$

Proof: Set

$$
q_{n}^{[\ell, 0]}(t)=\left|\begin{array}{ccc}
\pi_{n}\left(\mu_{1}\right) & \cdots & \pi_{n+\ell}\left(\mu_{1}\right) \\
\vdots & & \\
\pi_{n}\left(\mu_{\ell}\right) & \cdots & \pi_{n+\ell}\left(\mu_{\ell}\right) \\
\pi_{n}(t) & \cdots & \pi_{n+\ell}(t)
\end{array}\right| .
$$

We note that $q_{n}^{[\ell, 0]}(t)$ satisfies the condition $\int t^{j} q_{n}^{[\ell, 0]}(t) d \alpha(t)=0$ for all $j \in\{0, \ldots, n-1\}$. Also $q_{n}^{[\ell, 0]}\left(\mu_{j}\right)=0, j=1, \ldots, \ell$, and so $q_{n}^{[\ell, 0]}(t) /\left[\left(\mu_{1}-t\right) \cdots\left(\mu_{\ell}-t\right)\right]$ is a polynomial of degree at most $n$. Now observe that

$$
\int t^{j}\left[\frac{q_{n}^{[\ell, 0]}(t)}{\left(\mu_{1}-t\right) \cdots\left(\mu_{\ell}-t\right)}\right] d \alpha^{[\ell, 0]}(t)=0, \quad 0 \leqslant j<n,
$$

which means that $q_{n}^{[\ell, 0]}(t)$ divided by the product $\left(\mu_{1}-t\right) \cdots\left(\mu_{\ell}-t\right)$ is proportional to the $n$th monic orthogonal polynomial $\pi_{n}^{[\ell, 0]}(t)$ associated with the new measure $d \alpha^{[\ell, 0]}(t)$. Now $q_{n}^{[\ell, 0]}$ $\times(t)$ cannot vanish for any $t=\mu_{\ell+1}>Q, \mu_{\ell+1} \notin\left\{\mu_{1}, \ldots, \mu_{\ell}\right\}$. Indeed, if $q_{n}^{[\ell, 0]}\left(\mu_{\ell+1}\right)=0$, then there exist $\left\{\alpha_{i}\right\}_{i=0}^{\ell}$, not all zero, such that $p(t) \equiv \sum_{i=0}^{\ell} \alpha_{i} \pi_{n+i}(t)$ vanishes at $\left\{\mu_{i}\right\}_{i=1}^{\ell+1}$. Thus $\tilde{p}(t) \equiv p(t) / \Pi_{i=1}^{\ell+1}\left(\mu_{i}-t\right)$ is a polynomial of order $<n$, and as above, $\tilde{p}(t)$ is orthogonal to $t^{j}$, $0 \leqslant j<n$, with respect to the measure $d \alpha^{[\ell+1,0]}(t)$. Thus $\widetilde{p}(t) \equiv 0$ and hence $\alpha_{0}=\cdots=\alpha_{\ell}=0$, which is a contradiction. Replacing $\ell$ by $\ell-1$, we conclude that

$$
\left|\begin{array}{ccc}
\pi_{n}\left(\mu_{1}\right) & \cdots & \pi_{n+\ell-1}\left(\mu_{1}\right) \\
\vdots & & \\
\pi_{n}\left(\mu_{\ell}\right) & \cdots & \pi_{n+\ell-1}\left(\mu_{\ell}\right)
\end{array}\right| \neq 0 .
$$

Taking the limit $t \rightarrow \infty$ and noting that the coefficient of the highest degree of $\pi_{n}^{[\ell, 0]}(t)$ should be equal to 1 , we find the coefficient of proportionality and establish formula (2.1).

Representation (2.1) for the monic orthogonal polynomials associated with the measure $d \alpha^{[\ell, 0]}(t)$ immediately leads to the following result:

Corollary 2.2: The product of monic orthogonal polynomials $\Pi_{j=0}^{\ell} \pi_{n}^{[j, 0]}\left(\mu_{j+1}\right)$ defined with respect to the different measures $d \alpha^{[j, 0]}(t) \equiv\left(\mu_{j}-t\right) \cdots\left(\mu_{1}-t\right) d \alpha(t)$ is given by the formula

$$
\prod_{j=0}^{\ell} \pi_{n}^{[j, 0]}\left(\mu_{j+1}\right)=\frac{1}{\Delta(\mu)}\left|\begin{array}{ccc}
\pi_{n}\left(\mu_{1}\right) & \cdots & \pi_{n+\ell}\left(\mu_{1}\right) \\
\vdots & & \\
\pi_{n}\left(\mu_{\ell+1}\right) & \cdots & \pi_{n+\ell}\left(\mu_{\ell+1}\right)
\end{array}\right|,
$$

where $\Delta(\mu)=\Pi_{\ell+1 \geqslant i>j \geqslant 1}\left(\mu_{i}-\mu_{j}\right)$.

We observe that Corollary 2.2 gives the identity for the average of products of random characteristic polynomials obtained first by Brezin and Hikami. ${ }^{3}$

Theorem 2.3: Let $D_{N}[\mu, H]$ be the characteristic polynomial of the Hermitian matrix $H$. The following identity is valid: 


$$
\left\langle\prod_{j=1}^{L} D_{N}\left[\mu_{j}, H\right]\right\rangle_{\alpha}=\frac{1}{\Delta(\mu)}\left|\begin{array}{ccc}
\pi_{N}\left(\mu_{1}\right) & \cdots & \pi_{N+L-1}\left(\mu_{1}\right) \\
\vdots & & \\
\pi_{N}\left(\mu_{L}\right) & \cdots & \pi_{N+L-1}\left(\mu_{L}\right)
\end{array}\right|
$$

where the average is defined by (1.2).

Proof: To prove formula (2.6) we use the representation for the monic orthogonal polynomials in the case $L=1$ given in (1.5),

$$
\pi_{N}(\mu)=\frac{1}{Z_{N}} \int \cdots \int \prod_{i=1}^{N}\left(\mu-x_{i}\right) \Delta^{2}(x) d \alpha(x)
$$

Let $Z_{N}^{[\ell, 0]}$ be defined by

$$
Z_{N}^{[\ell, 0]}=\int \cdots \int \Delta^{2}(x) d \alpha^{[\ell, 0]}(x), \quad \ell=1,2, \ldots
$$

where $d \alpha^{[\ell, 0]}(x)=\prod_{i=1}^{N} d \alpha^{[\ell, 0]}\left(x_{i}\right)$. With this notation, we have

$$
\left\langle\prod_{j=1}^{L} D_{N}\left[\mu_{j}, H\right]\right\rangle_{\alpha}=\frac{Z_{N}^{[L, 0]}}{Z_{N}}=\frac{Z_{N}^{[L, 0]}}{Z_{N}^{[L-1,0]}} \frac{Z_{N}^{[L-1,0]}}{Z_{N}^{[L-2,0]}} \cdots \frac{Z_{N}^{[1,0]}}{Z_{N}} .
$$

Equation (2.7) implies that $\pi_{n}^{[\ell-1,0]}\left(\mu_{\ell}\right)$ can be represented as the ratio $Z_{N}^{[\ell, 0]} / Z_{N}^{[\ell-1,0]}$, where $\pi_{N}^{[0,0]}(\mu) \equiv \pi_{N}(\mu)$, and $Z_{N}^{[0,0]} \stackrel{n}{\equiv} Z_{N}$. Thus we obtain

$$
\left\langle\prod_{j=1}^{L} D_{N}\left[\mu_{j}, H\right]\right\rangle_{\alpha}=\prod_{j=0}^{L-1} \pi_{N}^{[j, 0]}\left(\mu_{j+1}\right)
$$

The above equation together with Corollary 2.2 proves formula (2.6).

Remark 2.4: Notice [see Eqs. (2.7) and (2.10)] that the average of products of characteristic polynomials can be rewritten as a product of averages. Namely,

$$
\left\langle\prod_{j=1}^{L} D_{N}\left[\mu_{j}, H\right]\right\rangle_{\alpha}=\prod_{j=1}^{L}\left\langle D_{N}\left[\mu_{j}, H\right]\right\rangle_{\alpha[j-1,0]}
$$

where $\langle\cdots\rangle_{\alpha[j-1,0]}$ means the average defined by Eq. (1.2) but with respect to the new measure $d \alpha^{[j-1,0]}(x)$, and $d \alpha(x) \equiv d \alpha^{[0,0]}(x)$.

The formula of Christoffel [Eq. (2.1)] enables us to construct the orthogonal polynomials associated with the measure $d \alpha^{[\ell, 0]}(t)=\prod_{j=1}^{\ell}\left(\mu_{j}-t\right) d \alpha(t)$ in terms of the orthogonal polynomials associated with the measure $d \alpha(t)$. Now we derive a formula due to Uvarov ${ }^{19}$ expressing the monic orthogonal polynomials $\pi_{n}^{[0, m]}(t)$ associated with the measure $d \alpha^{[0, m]}(t)=\Pi_{j=1}^{m}\left(\epsilon_{j}\right.$ $-t)^{-1} d \alpha(t)$, again in terms of the monic orthogonal polynomials $\pi_{n}(t)$ associated with the measure $d \alpha(t)$.

Lemma 2.5: Suppose $0 \leqslant m \leqslant n$. The monic orthogonal polynomials $\pi_{n}^{[0, m]}(t)$ associated with the measure $d \alpha^{[0, m]}(t)$ can be expressed as ratios of determinants. 


$$
\pi_{n}^{[0, m]}(t)=\frac{\left|\begin{array}{ccc}
h_{n-m}\left(\epsilon_{1}\right) & \cdots & h_{n}\left(\epsilon_{1}\right) \\
\vdots & & \\
h_{n-m}\left(\epsilon_{m}\right) & \cdots & h_{n}\left(\epsilon_{m}\right) \\
\pi_{n-m}(t) & \cdots & \pi_{n}(t)
\end{array}\right|}{\left|\begin{array}{ccc}
h_{n-m}\left(\epsilon_{1}\right) & \cdots & h_{n-1}\left(\epsilon_{1}\right) \\
\vdots & & \\
h_{n-m}\left(\epsilon_{m}\right) & \cdots & h_{n-1}\left(\epsilon_{m}\right)
\end{array}\right|} .
$$

Here the $h_{k}\left(\epsilon_{j}\right)$ 's are the Cauchy transformations of the monic orthogonal polynomials $\pi_{k}(t)$.

$$
h_{k}\left(\epsilon_{j}\right)=\frac{1}{2 \pi i} \int \frac{\pi_{k}(t) d \alpha(t)}{t-\epsilon_{j}} .
$$

Proof: Set

$$
q_{n}^{[0, m]}(t)=\left|\begin{array}{ccc}
h_{n-m}\left(\epsilon_{1}\right) & \cdots & h_{n}\left(\epsilon_{1}\right) \\
\vdots & & \\
h_{n-m}\left(\epsilon_{m}\right) & \cdots & h_{n}\left(\epsilon_{m}\right) \\
\pi_{n-m}(t) & \cdots & \pi_{n}(t)
\end{array}\right| .
$$

Now $q_{n}^{[0, m]}(t)$ is proportional to the $n$th monic orthogonal polynomial $\pi_{n}^{[0, m]}(t)$ with respect to the measure $d \alpha^{[0, m]}(t)$. Indeed, first observe that

$$
\int \frac{q_{n}^{[0, m]}(t)}{t-\boldsymbol{\epsilon}_{j}} d \alpha(t)=0, \quad j=1, \ldots, m .
$$

Also, for $0 \leqslant k<n$,

$$
\frac{t^{k}}{\Pi_{\ell=1}^{m}\left(\epsilon_{\ell}-t\right)}=\sum_{\ell=1}^{m} \frac{\beta_{\ell}}{\epsilon_{\ell}-t}+p(t)
$$

for suitable constants $\left\{\beta_{\ell}\right\}$ and for some polynomial $p(t)$ of degree $<n-m$. But for $0 \leqslant k<n$,

$$
\int t^{k} q_{n}^{[0, m]}(t) d \alpha^{[0, m]}(t)=-\sum_{\ell=1}^{m} \beta_{\ell} \int \frac{q_{n}^{[0, m]}(t)}{t-\epsilon_{\ell}} d \alpha(t)+\int p(t) q_{n}^{[0, m]}(t) d \alpha(t) .
$$

The terms in the sum are zero by (2.15) and the final integral is zero by the construction (2.14) of $q_{n}^{[0, m]}(t)$ and the fact that $\operatorname{deg} p(t)<n-m$. Thus $q_{n}^{[0, m]}(t)$ is proportional to $\pi_{n}^{[0, m]}(t)$. An argument similar to the proof in Lemma 2.1, that

$$
\left|\begin{array}{ccc}
\pi_{n}\left(\mu_{1}\right) & \cdots & \pi_{n+\ell-1}\left(\mu_{1}\right) \\
\vdots & & \\
\pi_{n}\left(\mu_{\ell}\right) & \cdots & \pi_{n+\ell-1}\left(\mu_{\ell}\right)
\end{array}\right| \neq 0,
$$

shows that the denominator in (2.12) does not vanish. Letting $t \rightarrow \infty$ in (2.14), and matching leading terms, we prove Lemma 2.5 .

Remark 2.6: In Ref. 19, Uvarov obtains formulas for $\pi_{n}^{[0, m]}(t)$ of type (2.12) also in the case $m>n$. These formulas can be used to obtain analogs of (2.24) and (2.36) below in the case $M$ $>N$. 
Remark 2.7: As noted in Refs. 11 and 17, the Cauchy transformations $h_{k}(\epsilon)$ of the $\pi_{k}$ 's occur explicitly, together with the $\pi_{k}$ 's, in the solution of the Fokas-Its-Kitaev Riemann-Hilbert problem for orthogonal polynomials. ${ }^{9}$

Lemma 2.5 implies the following analog of the Christoffel formula for the Cauchy transforms of monic orthogonal polynomials.

Corollary 2.8: Let $h_{k}^{[0, m]}(\epsilon)$ be the Cauchy transform of the monic polynomial $\pi_{k}^{[0, m]}(t)$. with respect to the measure $d \alpha^{[0, m]}(t)$,

$$
h_{k}^{[0, m]}(\epsilon)=\frac{1}{2 \pi i} \int \frac{\pi_{k}^{[0, m]}(t)}{t-\epsilon} d \alpha^{[0, m]}(t) .
$$

Let also $0 \leqslant m \leqslant n$. Then $h_{n}^{[0, m]}(\epsilon)$ has a representation similar to that for the monic orthogonal polynomials $\pi_{n}^{[\ell, 0]}(t)$ [Eq. (2.1)],

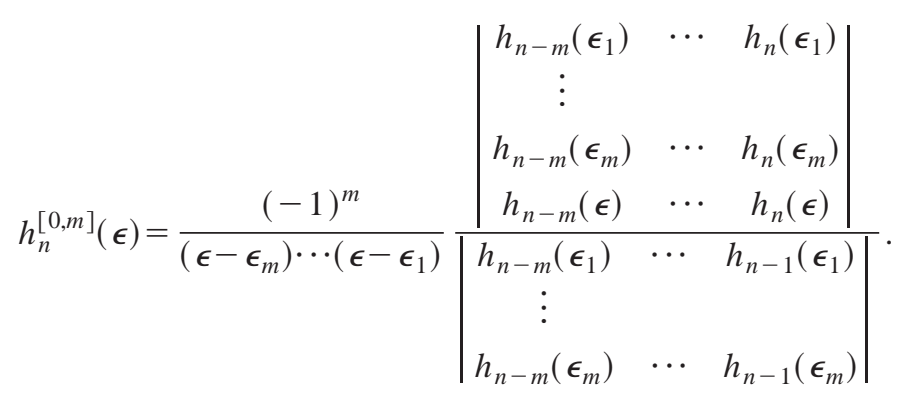

Proof: The above representation follows from formula (2.12) and from the fact that

$$
\frac{1}{\left(t-\epsilon_{m+1}\right) \cdots\left(t-\epsilon_{1}\right)}=\sum_{j=1}^{m+1} \frac{1}{t-\epsilon_{j}} \prod_{k \neq j} \frac{1}{\epsilon_{j}-\epsilon_{k}} .
$$

Indeed we find from formula $(2.12)$ that $h_{n}^{[0, m]}(\epsilon)$ is the ratio of the determinants. The elements of the last row of the determinant in the numerator are the integrals

$$
\frac{1}{2 \pi i} \int \frac{\pi_{n-k}(t) d \alpha(t)}{(t-\epsilon)\left(t-\epsilon_{m}\right) \cdots\left(t-\epsilon_{1}\right)}, \quad 0 \leqslant k \leqslant m .
$$

Using identity (2.21) and noting that the only term

$$
\frac{1}{t-\epsilon} \frac{1}{\left(\epsilon-\epsilon_{m}\right) \cdots\left(\epsilon-\epsilon_{1}\right)}
$$

of the sum (2.21) contributes to the determinant, (2.20) follows.

Equation (2.20) immediately implies the following analogy of (2.5) for the $h_{k}^{[0, m]}$,s.

Corollary 2.9: Let $0 \leqslant m \leqslant n$. Then the product of the Cauchy transforms of monic orthogonal polynomials with respect to the measures $d \alpha^{[0, j]}(t), 0 \leqslant j \leqslant m$, can be written as a determinant,

$$
\prod_{j=0}^{m} h_{n-m+j}^{[0, j]}\left(\epsilon_{j+1}\right)=\frac{(-1) m(m+1) / 2}{\Delta(\epsilon)}\left|\begin{array}{ccc}
h_{n-m}\left(\epsilon_{1}\right) & \cdots & h_{n}\left(\epsilon_{1}\right) \\
\vdots & & \\
h_{n-m}\left(\epsilon_{m+1}\right) & \cdots & h_{n}\left(\epsilon_{m+1}\right)
\end{array}\right| .
$$

Now we derive the identity for the average of the product of inverse random characteristic polynomials.

Theorem 2.10: Suppose $1 \leqslant M \leqslant N$ and let $\gamma_{n}=-2 \pi i / c_{n}^{2}$, where $c_{n}$ is the normality constant defined by Eq. (1.4). Then we have the following formula: 


$$
\left\langle\prod_{j=1}^{M} D_{N}^{-1}\left[\epsilon_{j}, H\right]\right\rangle_{\alpha}=(-1)^{M(M-1) / 2} \frac{\prod_{j=N-M}^{N-1} \gamma_{j}}{\Delta(\epsilon)}\left|\begin{array}{ccc}
h_{N-M}\left(\epsilon_{1}\right) & \cdots & h_{N-1}\left(\epsilon_{1}\right) \\
\vdots & & \\
h_{N-M}\left(\epsilon_{M}\right) & \cdots & h_{N-1}\left(\epsilon_{M}\right)
\end{array}\right| .
$$

Proof: When $M=1$, we use the identity (2.21) together with (2.7) and the relation (see, e.g., Ref. 18)

$$
\gamma_{n-1}=-2 \operatorname{\pi in} \frac{Z_{n-1}}{Z_{n}}
$$

to obtain

$$
\left\langle D_{N}^{-1}[\epsilon, H]\right\rangle_{\alpha}=\gamma_{N-1} h_{N-1}(\epsilon) .
$$

We rewrite the average in Eq. (2.24) as follows:

$$
\left\langle\prod_{j=1}^{M} D_{N}^{-1}\left[\epsilon_{j}, H\right]\right\rangle_{\alpha}=\frac{Z_{N}^{[0, M]}}{Z_{N-1}^{[0, M-1]}} \frac{Z_{N-1}^{[0, M-1]}}{Z_{N-2}^{[0, M-2]}} \cdots \frac{Z_{N-M}^{[0,0]}}{Z_{N}^{[0,0]}},
$$

where

$$
Z_{N}^{[0, M]}=\int \cdots \int \Delta^{2}(x) d \alpha^{[0, M]}(x)
$$

$Z_{N}^{[0,0]} \equiv Z_{N}$ and $d \alpha^{[0,0]}(x)=d \alpha(x)$. The following relation can be observed from Eqs. (2.26) and (2.25):

$$
\frac{Z_{N-K}^{[0, m]}}{Z_{N-K-1}^{[0, m-1]}}=-2 \pi i(N-K) h_{N-K-1}^{[0, m-1]}\left(\epsilon_{m}\right) .
$$

Inserting this relation in (2.27) we find

$$
\left\langle\prod_{j=1}^{M} D_{N}^{-1}\left[\epsilon_{j}, H\right]\right\rangle_{\alpha}=\prod_{j=1}^{M} \gamma_{N-j} h_{N-j}^{[0, M-j]}\left(\epsilon_{M-j+1}\right) .
$$

Our result (2.24) immediately follows from the above equation and formula (2.23).

We now repeat the above considerations for the case

$$
d \alpha^{[\ell, m]}(t)=\frac{\left(\mu_{1}-t\right) \cdots\left(\mu_{\ell}-t\right)}{\left(\epsilon_{1}-t\right) \cdots\left(\epsilon_{m}-t\right)} d \alpha(t) .
$$

The first result is a Christoffel-type formula for the measure (2.31), which is due to Uvarov. ${ }^{19}$

Lemma 2.11: Suppose $0 \leqslant m \leqslant n$. Then the monic orthogonal polynomials $\pi_{n}^{[\ell, m]}(t)$ 's with respect to the measure $d \alpha^{\ell, m]}(t)$ have the following representation: 


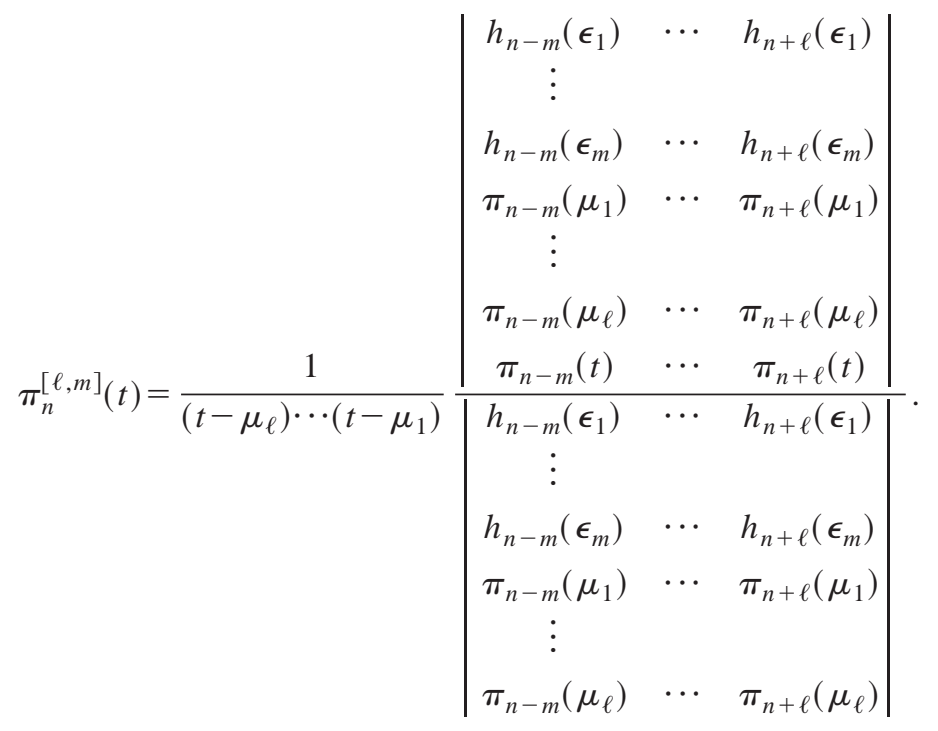

Proof: As in the previous cases we define $q_{n}^{[\ell, m]}(t)$ to be the determinant in the numerator of (2.32). Observe that

$$
q_{n}^{[\ell, m]}\left(\mu_{1}\right)=\cdots=q_{n}^{[\ell, m]}\left(\mu_{\ell}\right)=0
$$

and that

$$
\int \frac{q_{n}^{[\ell, m]}(t) d \alpha(t)}{\epsilon_{1}-t}=\cdots=\int \frac{q_{n}^{[\ell, m]}(t) d \alpha(t)}{\epsilon_{m}-t}=0 .
$$

The next steps are the same as in the proofs of Lemma 2.1 and Lemma 2.5.

Corollary 2.12:

$$
\left\langle\prod_{j=1}^{K} D_{N}\left[\mu_{j}, H\right]\right\rangle_{\alpha[0, M]}=\frac{1}{\Delta(\mu)} \frac{\left|\begin{array}{ccc}
h_{N-M}\left(\epsilon_{1}\right) & \cdots & h_{N+K-1}\left(\epsilon_{1}\right) \\
\vdots & & \\
h_{N-M}\left(\epsilon_{M}\right) & \cdots & h_{N+K-1}\left(\epsilon_{M}\right) \\
\pi_{N-M}\left(\mu_{1}\right) & \cdots & \pi_{N+K-1}\left(\mu_{1}\right) \\
\vdots & & \\
\pi_{N-M}\left(\mu_{K}\right) & \cdots & \pi_{N+K-1}\left(\mu_{K}\right)
\end{array}\right|}{\left|\begin{array}{cccc}
h_{N-M}\left(\epsilon_{1}\right) & \cdots & h_{N}\left(\epsilon_{1}\right) \mid \\
\vdots & & \\
\mid h_{N-M}\left(\epsilon_{M}\right) & \cdots & h_{N}\left(\epsilon_{M}\right)
\end{array}\right|} .
$$

Proof: Identity (2.35) follows from Eqs. (2.10) and (2.32) once we note that Eq. (2.32) can be rewritten in a similar manner as Eq. (2.5).

Finally we generalize Theorems 2.3 and 2.10 and obtain a formula for the average of ratios of characteristic polynomials.

Theorem 2.13: Suppose $0 \leqslant M \leqslant N$. Then the average of ratios of characteristic polynomials of $N \times N$ Hermitian matrices $H$ is given by the following formula: 


$$
\left|\frac{\prod_{j=1}^{K} D_{N}\left[\mu_{j}, H\right]}{\prod_{j=1}^{M} D_{N}\left[\epsilon_{j}, H\right]}\right|_{\alpha}=\frac{(-1)^{M(M-1) / 2} \prod_{j=N-M}^{N-1} \gamma_{j}}{\Delta(\mu) \Delta(\epsilon)}\left|\begin{array}{ccc}
h_{N-M}\left(\epsilon_{1}\right) & \cdots & h_{N+K-1}\left(\epsilon_{1}\right) \\
\vdots & & \\
h_{N-M}\left(\epsilon_{M}\right) & \cdots & h_{N+K-1}\left(\epsilon_{M}\right) \\
\pi_{N-M}\left(\mu_{1}\right) & \cdots & \pi_{N+K-1}\left(\mu_{1}\right) \\
\vdots & & \\
\pi_{N-M}\left(\mu_{K}\right) & \cdots & \pi_{N+K-1}\left(\mu_{K}\right)
\end{array}\right| .
$$

Proof: Let $\alpha^{[0,0]} \equiv \alpha, Z_{n}^{[0,0]} \equiv Z_{n}$. Then we have

$$
\left|\frac{\prod_{j=1}^{K} D_{N}\left[\mu_{j}, H\right]}{\prod_{j=1}^{M} D_{N}\left[\epsilon_{j}, H\right]}\right|_{\alpha}=\frac{Z_{N}^{[K, M]}}{Z_{N}^{[0,0]}}=\frac{Z_{N}^{[K, M]}}{Z_{N}^{[0, M]}} \frac{Z_{N}^{[0, M]}}{Z_{N}^{[0,0]}},
$$

i.e.,

$$
\left\langle\frac{\prod_{j=1}^{K} D_{N}\left[\mu_{j}, H\right]}{\prod_{j=1}^{M} D_{N}\left[\epsilon_{j}, H\right]}\right\rangle_{\alpha}=\left\langle\prod_{j=1}^{K} D_{N}\left[\mu_{j}, H\right]\right\rangle_{\alpha[0, M]}\left\langle\prod_{j=1}^{M} D_{N}^{-1}\left[\epsilon_{j}, H\right]\right\rangle_{\alpha} .
$$

We use Corollary 2.12 and Theorem 2.10 to obtain formula (2.36).

Remark 2.14: Observe that formulas (2.6) and (2.24) do not follow immediately as special cases of (2.36): some further algebraic manipulation is required. Similarly, the process of adding and removing zeros is clearly reciprocal. More precisely, given $\epsilon_{1}, \ldots, \epsilon_{\ell}$, we can construct the polynomials $\pi_{n}^{[0, \ell]}\left(t ; d \alpha^{[0, \ell]}\right)$ associated with the measure $d \alpha^{[0, \ell]}(t)=\left(\Pi_{i=1}^{\ell}\left(\epsilon_{i}-t\right)^{-1}\right) d t$ by (2.12): We can then construct $\pi_{n}^{[\ell, 0]}\left(t ; d\left(\alpha^{[0, \ell]}\right)^{[\ell, 0]}\right)$ with $\mu_{i}=\epsilon_{i}$, inserting $\pi_{n}^{[0, \ell]}\left(t ; d \alpha^{[0, \ell]}\right)$ for $\pi_{n}(t)$ on the right-hand side of $(2.1)$. We should find that $\pi_{n}^{[\ell, 0]}\left(t ; d\left(\alpha^{[0, \ell]}\right)^{[\ell, 0]}\right)=\pi_{n}(t ; d \alpha)$. However, again, this relation is not immediately clear, and requires further algebraic manipulation.

\section{FORMULAS OF TWO-POINT FUNCTION TYPE}

The following integral version of the Binet-Cauchy formula is due to Andréief, ${ }^{2}$ and plays a basic role in our calculations.

Lemma 3.1: Let $(X, d \mu)$ be a measure space and suppose $f_{i}, g_{j} \in L^{2}(X, d \mu)$ for $1 \leqslant i, j \leqslant k$. Then

$$
\begin{gathered}
\int_{X} \cdots \int_{X} \operatorname{det}\left(f_{i}\left(x_{j}\right)\right)_{1 \leqslant i, j \leqslant k} \operatorname{det}\left(g_{i}\left(x_{j}\right)\right)_{1 \leqslant i, j \leqslant k} d \mu\left(x_{1}\right) \cdots d \mu\left(x_{k}\right) \\
\quad=k ! \operatorname{det}\left(\int_{X} f_{i}(x) g_{j}(x) d \mu(x)\right)_{1 \leqslant i, j \leqslant k} .
\end{gathered}
$$

Proof: Set $c_{i j}=\int_{X} f_{i}(x) g_{j}(x) d \mu(x)$. Then

$$
\begin{aligned}
& \int_{X} \cdots \int_{X} \operatorname{det}\left(f_{i}\left(x_{j}\right)\right)_{1 \leqslant i, j \leqslant k} \operatorname{det}\left(g_{i}\left(x_{j}\right)\right)_{1 \leqslant i, j \leqslant k} d \mu\left(x_{1}\right) \cdots d \mu\left(x_{k}\right) \\
& =\sum_{\sigma, \tau \in S_{k}} \operatorname{sgn}(\sigma) \operatorname{sgn}(\tau) c_{\sigma(1) \tau(1)} \cdots c_{\sigma(k) \tau(k)}=\sum_{\sigma} \operatorname{sgn}(\sigma) \sum_{\tau} \operatorname{sgn}\left(\tau^{\circ} \sigma\right) c_{\sigma(1) \tau^{\circ} \sigma(1)} \cdots c_{\sigma(k) \tau^{\circ} \sigma(k)} \\
& =\sum_{\sigma}(\operatorname{sgn}(\sigma))^{2} \sum_{\tau} \operatorname{sgn}(\tau) c_{1 \tau(1)} \cdots c_{k \tau(k)}=k ! \operatorname{det}\left(c_{i j}\right)_{1 \leqslant i, j \leqslant k}
\end{aligned}
$$


as desired. In (3.2) we used $\operatorname{sgn}\left(\tau^{\circ} \sigma\right)=(\operatorname{sgn} \tau)(\operatorname{sgn} \sigma)$ and the fact that $c_{\sigma(1) \tau^{\circ} \sigma(1)} \cdots c_{\sigma(k) \tau^{\circ} \sigma(k)}$ $=c_{1 \tau(1)}{ }^{\cdots} c_{k \tau(k)}$ for all $\sigma$.

Theorem 3.2: Let $K \geqslant 1$. Then the following identity is valid:

$$
\left\langle\prod_{j=1}^{K} D_{N}\left[\lambda_{j}, H\right] D_{N}\left[\mu_{j}, H\right]\right\rangle_{\alpha}=\frac{C_{N, K}}{\Delta(\lambda) \Delta(\mu)} \operatorname{det}\left(W_{I, N+K}\left(\lambda_{i}, \mu_{j}\right)\right)_{1 \leqslant i, j \leqslant K},
$$

where

$$
W_{I, N+K}(x, y)=\frac{\pi_{N+K}(x) \pi_{N+K-1}(y)-\pi_{N+K}(y) \pi_{N+K-1}(y)}{x-y}
$$

and

$$
C_{N, K}=\frac{\prod_{\ell=N}^{N+K-1} c_{\ell}^{2}}{\left(c_{N+K-1}\right)^{2 K}}
$$

where $c_{\ell}$ is again the norming constant for $\pi_{\ell}$ given in (1.4).

Proof: Let $p_{j}(x)=c_{j}^{-1} \pi_{j}(x), j \geqslant 0$, denote the orthonormal polynomials with respect to $d \alpha$. From (1.2) we obtain

$$
\left\langle\prod_{j=1}^{K} D_{N}\left[\lambda_{j}, H\right] D_{N}\left[\mu_{j}, H\right]\right\rangle_{\alpha}=\frac{1}{Z_{N} \Delta(\lambda) \Delta(\mu)} \int \cdots \int \Delta(x, \lambda) \Delta(x, \mu) d \alpha(x) .
$$

Adding columns, we see that the Vandermonde determinant $\Delta(x, \lambda)$ has the form

$$
\left|\begin{array}{cccc}
\pi_{0}\left(x_{1}\right) & \pi_{1}\left(x_{1}\right) & \cdots & \pi_{N+K-1}\left(x_{1}\right) \\
\vdots & & & \\
\pi_{0}\left(x_{N}\right) & \pi_{1}\left(x_{N}\right) & \cdots & \pi_{N+K-1}\left(x_{N}\right) \\
\pi_{0}\left(\lambda_{1}\right) & \pi_{1}\left(\lambda_{1}\right) & \cdots & \pi_{N+K-1}\left(\lambda_{1}\right) \\
\vdots & & & \\
\pi_{0}\left(\lambda_{K}\right) & \pi_{1}\left(\lambda_{K}\right) & \cdots & \pi_{N+K-1}\left(\lambda_{K}\right)
\end{array}\right|
$$

and similarly for $\Delta(x, \mu)$. Here $\pi_{j}(t)=\pi_{j}^{[0,0]}(t)$. The determinant $\Delta(x, \lambda)$ can be evaluated by a Lagrange expansion of the form

$$
\sum_{0 \leqslant i_{1}<i_{2}<\cdots<i_{k} \leqslant N+K-1} \sigma_{i_{1}, \ldots, i_{K}}\left|\begin{array}{ccc}
\pi_{i_{1}}\left(\lambda_{1}\right) & \cdots & \pi_{i_{K}}\left(\lambda_{1}\right) \\
\vdots & & \\
\pi_{i_{1}}\left(\lambda_{K}\right) & \cdots & \pi_{i_{K}}\left(\lambda_{K}\right)
\end{array}\right|\left|\begin{array}{ccc}
\pi_{j_{1}}\left(x_{1}\right) & \cdots & \pi_{j_{N}}\left(x_{1}\right) \\
\vdots & & \\
\pi_{j_{1}}\left(x_{N}\right) & \cdots & \pi_{j_{N}}\left(x_{N}\right)
\end{array}\right|,
$$

where $\sigma_{i_{1}, \ldots, i_{K}}= \pm 1$ is an appropriate signature and $\left\{\left(j_{1}, \ldots, j_{N}\right): 0 \leqslant j_{1}<j_{2}<\cdots<j_{N} \leqslant N+K\right.$ $-1\}$ is the complement of $\left\{i_{1}, \ldots, i_{K}\right\}$ in $\{0,1, \ldots, N+K-1\}$. Multiplying (3.8) by a similar expansion for $\Delta(x, \mu)$, and inserting in (3.6), we obtain a sum of terms of the form

$$
\int \cdots \int\left|\begin{array}{ccc}
\pi_{j_{1}}\left(x_{1}\right) & \cdots & \pi_{j_{K}}\left(x_{1}\right) \\
\vdots & & \\
\pi_{j_{1}}\left(x_{N}\right) & \ldots & \pi_{j_{K}}\left(x_{N}\right)
\end{array}\right|\left|\begin{array}{ccc}
\pi_{j_{1}^{\prime}}\left(x_{1}\right) & \ldots & \pi_{j_{N}^{\prime}}\left(x_{1}\right) \\
\vdots & & \\
\pi_{j_{1}^{\prime}}\left(x_{N}\right) & \ldots & \pi_{j_{N}^{\prime}}\left(x_{N}\right)
\end{array}\right| d \alpha(x)
$$


which is equal by Lemma 3.1 to $N ! \operatorname{det}\left(\int \pi_{j_{i}^{\prime}}(x) \pi_{j_{k}}(x) d \alpha(x)\right)_{1 \leqslant i, k \leqslant N}=N ! \operatorname{det}\left(\delta_{j_{i}^{\prime} j_{k}} c_{j_{k}}^{2}\right)_{1 \leqslant i, k \leqslant N}$. From this we see that

$$
\begin{aligned}
& \left\langle\prod_{j=1}^{K} D_{N}\left[\lambda_{j}, H\right] D_{N}\left[\mu_{j}, H\right]\right\rangle_{\alpha} \\
& =\frac{N !}{Z_{N} \Delta(\lambda) \Delta(\mu)} \sum_{0 \leqslant i_{1}<\cdots<i_{k} \leqslant N+K-1} \sigma_{i_{1}, \ldots, i_{K}}^{2}\left|\begin{array}{ccc}
\pi_{i_{1}}\left(\lambda_{1}\right) & \cdots & \pi_{i_{K}}\left(\lambda_{1}\right) \\
\vdots & & \\
\pi_{i_{1}}\left(\lambda_{K}\right) & \cdots & \pi_{i_{K}}\left(\lambda_{K}\right)
\end{array}\right| \\
& \times \prod_{k=1}^{N} c_{j_{k}}^{2}\left|\begin{array}{ccc}
\pi_{i_{1}}\left(\mu_{1}\right) & \cdots & \pi_{i_{K}}\left(\mu_{1}\right) \\
\vdots & & \\
\pi_{i_{1}}\left(\mu_{K}\right) & \cdots & \pi_{i_{K}}\left(\mu_{K}\right)
\end{array}\right| \\
& =\frac{N ! \Pi_{q=N}^{N+K-1} c_{q}^{2}}{Z_{N} \Delta(x, \lambda) \Delta(x, \mu)} \sum_{0 \leqslant i_{1}<\cdots<i_{k} \leqslant N+K-1} \operatorname{det}\left(p_{i_{j}}\left(\lambda_{k}\right)\right)_{1 \leqslant j, k \leqslant K} \operatorname{det}\left(p_{i_{j}}\left(\mu_{k}\right)\right)_{1 \leqslant j, k \leqslant K} \\
& =\frac{N ! \Pi_{q=N}^{N+K-1} c_{q}^{2}}{Z_{N} \Delta(x, \lambda) \Delta(x, \mu)} \operatorname{det}\left(\sum_{0 \leqslant i \leqslant N+K-1} p_{i}\left(\lambda_{j}\right) p_{i}\left(\mu_{k}\right)\right)_{1 \leqslant j, k \leqslant K},
\end{aligned}
$$

where the last line follows by applying Lemma 3.1 to the discrete measure $d \mu=\sum_{i=0}^{N+K-1} \delta_{i}$. But, by the Christoffel-Darboux formula,

$$
\sum_{0 \leqslant i \leqslant N+K-1} p_{i}\left(\lambda_{j}\right) p_{i}\left(\mu_{k}\right)=\frac{\pi_{N+K}\left(\lambda_{j}\right) \pi_{N+K-1}\left(\mu_{k}\right)-\pi_{N+K}\left(\mu_{k}\right) \pi_{N+K-1}\left(\lambda_{j}\right)}{\lambda_{j}-\mu_{k}},
$$

which then implies (3.3) as $Z_{N}=N ! \Pi_{\ell=0}^{N-1} c_{\ell}^{2}$ (see, e.g., Ref. 18).

Theorem 3.3: Suppose $1 \leqslant K \leqslant N$. Then the following identity is valid:

$$
\left\langle\prod_{j=1}^{K} \frac{D_{N}\left[\mu_{i}, H\right]}{D_{N}\left[\epsilon_{j}, H\right]}\right\rangle_{\alpha}=(-1)^{K(K-1) / 2} \gamma_{N-1}^{K} \frac{\Delta(\epsilon, \mu)}{\Delta^{2}(\epsilon) \Delta^{2}(\mu)} \operatorname{det}\left(W_{H, N}\left(\epsilon_{i}, \mu_{j}\right)\right)_{1 \leqslant i, j \leqslant K},
$$

where

$$
W_{H, N}(x, y)=\frac{h_{N}(\epsilon) \pi_{N-1}(\mu)-h_{N-1}(\epsilon) \pi_{N}(\mu)}{\epsilon-\mu}
$$

and again $h_{k}(\epsilon)=(1 / 2 \pi i) \int \pi_{k}(t) d \alpha(t) /(t-\epsilon)$ is the Cauchy transform of $\pi_{k}(t)$ and $\gamma_{N-1}=$ $-2 \pi i / C_{N-1}^{2}$. Observe first that by linearity 


$$
\begin{aligned}
& \left|\begin{array}{ccc}
h_{N-M}\left(\epsilon_{1}\right) & \cdots & h_{N+L-1}\left(\epsilon_{1}\right) \\
\vdots & & \\
h_{N-M}\left(\epsilon_{M}\right) & \cdots & h_{N+L-1}\left(\epsilon_{M}\right) \\
\pi_{N-M}\left(\mu_{1}\right) & \cdots & \pi_{N+L-1}\left(\mu_{1}\right) \\
\vdots & & \\
\pi_{N-M}\left(\mu_{1}\right) & \cdots & \pi_{N+L-1}\left(\mu_{L}\right)
\end{array}\right|=\int \cdots \int \frac{d \alpha(\lambda)}{(2 \pi i)^{M} \prod_{j=1}^{M}\left(\lambda_{j}-\epsilon_{j}\right)} \\
& \times\left|\begin{array}{ccc}
\pi_{N-M}\left(\lambda_{1}\right) & \cdots & \pi_{N+L-1}\left(\lambda_{1}\right) \\
\vdots & & \\
\pi_{N-M}\left(\lambda_{M}\right) & \cdots & \pi_{N+L-1}\left(\lambda_{M}\right) \\
\pi_{N-M}\left(\mu_{1}\right) & \cdots & \pi_{N+L-1}\left(\mu_{1}\right) \\
\vdots & & \\
\pi_{N-M}\left(\mu_{1}\right) & \cdots & \pi_{N+L-1}\left(\mu_{L}\right)
\end{array}\right|
\end{aligned}
$$

Inserting (2.36) on the left-hand side, and using (2.5) to reexpress the integrand on the right-hand side, we obtain the following result, which is of independent interest. The result expresses averages of ratios of characteristic polynomials in terms of averages of products of such polynomials.

Proposition 3.4: Let $1 \leqslant M \leqslant N$. Then

$$
\begin{aligned}
\left|\frac{\prod_{j=1}^{L} D_{N}\left[\mu_{i}, H\right]}{\prod_{j=1}^{M} D_{N}\left[\epsilon_{j}, H\right]}\right|_{\alpha} & \frac{(-1)^{M(M-1) / 2} \prod_{j=N-M}^{N-1} \gamma_{j}}{\Delta(\mu) \Delta(\epsilon)} \int \cdots \int \frac{d \alpha(\lambda)}{(2 \pi i)^{M} \prod_{j=1}^{M}\left(\lambda_{j}-\epsilon_{j}\right)} \Delta(\lambda, \mu) \\
& \times\left\langle\prod_{j=1}^{M} D_{N-M}\left[\lambda_{j}, H\right] \prod_{j=1}^{L} D_{N-M}\left[\mu_{j}, H\right]\right\rangle_{\alpha} .
\end{aligned}
$$

Proof of Theorem 3.2: For $M=L=K \leqslant N$, by (3.15) and (3.3),

$$
\begin{aligned}
& \frac{\Delta(\mu) \Delta(\epsilon)}{(-1)^{K(K-1) / 2} \prod_{j=N-K}^{N-1} \gamma_{j}}\left\langle\left.\frac{\prod_{j=1}^{K} D_{N}\left[\mu_{i}, H\right]}{\prod_{j=1}^{K} D_{N}\left[\epsilon_{j}, H\right]}\right|_{\alpha}\right. \\
& =\int \cdots \int \frac{d \alpha(\lambda)}{(2 \pi i)^{M} \prod_{j=1}^{M}\left(\lambda_{j}-\epsilon_{j}\right)} C_{N-K, K} \prod_{i=1}^{K} \prod_{j=1}^{K}\left(\mu_{i}-\lambda_{j}\right) \operatorname{det}\left(W_{I, N}\left(\lambda_{i}, \mu_{j}\right)\right)_{1 \leqslant i, j \leqslant K} .
\end{aligned}
$$

But

$$
\begin{aligned}
& \frac{1}{2 \pi i} \int \frac{d \alpha\left(\lambda_{j}\right)}{\lambda_{j}-\epsilon_{j}} \prod_{i=1}^{K}\left(\mu_{i}-\lambda_{j}\right) \frac{\pi_{N}\left(\lambda_{j}\right) \pi_{N-1}\left(\mu_{k}\right)-\pi_{N-1}\left(\lambda_{j}\right) \pi_{N}\left(\mu_{k}\right)}{\lambda_{j}-\mu_{k}} \\
& =\frac{1}{2 \pi i} \int d \alpha\left(\lambda_{j}\right)\left(1-\frac{\mu_{1}-\epsilon_{j}}{\lambda_{j}-\epsilon_{j}}\right)\left(\prod_{\substack{i=2 \\
i \neq k}}^{K}\left(\mu_{i}-\lambda_{j}\right)\right)\left(\pi_{N}\left(\lambda_{j}\right) \pi_{N-1}\left(\mu_{k}\right)-\pi_{N-1}\left(\lambda_{j}\right) \pi_{N}\left(\mu_{k}\right)\right) \\
& =-\frac{1}{2 \pi i} \int d \alpha\left(\lambda_{j}\right) \frac{\mu_{1}-\epsilon_{j}}{\lambda_{j}-\epsilon_{j}}\left(\prod_{\substack{i=2 \\
i \neq k}}^{K}\left(\mu_{i}-\lambda_{j}\right)\right)\left(\pi_{N}\left(\lambda_{j}\right) \pi_{N-1}\left(\mu_{k}\right)-\pi_{N-1}\left(\lambda_{j}\right) \pi_{N}\left(\mu_{k}\right)\right)
\end{aligned}
$$


as $\int d \alpha\left(\lambda_{j}\right) \lambda_{j}^{\ell} \pi_{N-1}\left(\lambda_{j}\right)=\int d \alpha\left(\lambda_{j}\right) \lambda_{j}^{\ell} \pi_{N}\left(\lambda_{j}\right)=0$ for $0 \leqslant \ell \leqslant K-2<N-1$. Continuing in this way, the integral reduces to $\Pi_{i=1}^{K}\left(\mu_{i}-\epsilon_{j}\right) W_{H, N}\left(\epsilon_{i}, \mu_{k}\right)$. Thus we find

$$
\frac{\Delta(\mu) \Delta(\epsilon)}{(-1)^{K(K-1) / 2} \prod_{j=N-K}^{N-1} \gamma_{j}}\left|\frac{\prod_{j=1}^{K} D_{N}\left[\mu_{i}, H\right]}{\prod_{j=1}^{K} D_{N}\left[\epsilon_{j}, H\right]}\right|_{\alpha}=\frac{\Delta(\epsilon, \mu)}{\Delta(\epsilon) \Delta(\mu)} \operatorname{det}\left(W_{I, N+K}\left(\lambda_{i}, \mu_{k}\right)\right)_{1 \leqslant i, k \leqslant K}
$$

and (3.12) follows.

\section{ACKNOWLEDGMENTS}

The authors would like to thank Jeff Geronimo for useful conversations and for pointing out the paper of U. B. Uvarov. The authors would also like to thank Nick Witte for many useful remarks. The work of the first author was supported in part by NSF Grant No. DMS-0208577. The work of the second author was supported in part by NSF Grant No. DMS-0296084 and by the Institute for Advanced Study in Princeton. The work of the third author was supported in part by EPSRC Grant No. GR/13838/01 "Random Matrices close to Unitary or Hermitian" and by the Brunel University Vice-Chancellor grant.

${ }^{1}$ Andreev, A. V. and Simons, V. D., "Correlators of spectral determinants in quantum chaos," Phys. Rev. Lett. 75 , 2304-2307 (1995).

${ }^{2}$ Andréief, C., "Note sur une relation les intégrales définies des produits des fonctions," Mém. Soc. Sci. Bordeaux 2, 1-14 (1883).

${ }^{3}$ Brezin, E. and Hikami, S., "Characteristic polynomials of random matrices," Commun. Math. Phys. 214, 111-135 (2000).

${ }^{4}$ Deift, P., Orthogonal Polynomials and Random Matrices: a Riemann-Hilbert Approach, Vol. 3 of Courant Lecture Notes in Mathematics (AMS, Providence, 2000).

${ }^{5}$ Deift, P. and Zhou, X., "A steepest descent method for oscillatory Riemman-Hilbert problems. Asymptotics for the MKdV equation," Ann. Math. 137, 295-368 (1993).

${ }^{6}$ Deift, P., Venakides, S., and Zhou, X., "New results in small dispersion KdV by an extension of the steepest descent method for Riemann-Hilbert problems," Int. Math. Res. Notices 6, 285-299 (1997).

${ }^{7}$ Deift, P., Kriecherbauer, T., McLaughlin, K., Venakides, S., and Zhou, X., "Uniform asymptotics for polynomials orthogonal with respect to varying exponential weights and applications to universality questions in random matrix theory," Commun. Pure Appl. Math. 52, 1335-1425 (1999).

${ }^{8}$ Deift, P., Kriecherbauer, T., McLaughlin, K., Venakides, S., and Zhou, X., "Strong asymptotics of orthogonal polynomials with respect to exponential weights," Commun. Pure Appl. Math. 52, 1491-1552 (1999).

${ }^{9}$ Fokas, A., Its, A., and Kitaev, V., "Discrete Painlevé equations and their appearance in quantum gravity," Commun. Math. Phys. 142, 313-344 (1991).

${ }^{10}$ Forrester, P. and Witte, N., "Applications of the tau-function theory of Painlevé equations to random matrices: PIV, PII and the GUE," Commun. Math. Phys. 219, 357-398 (2001).

${ }^{11}$ Fyodorov, Y. V. and Strahov, E., "An exact formula for general spectral correlation functions of random matrices," J. Phys. A 36, 3203-3213 (2003).

${ }^{12}$ Hughes, C., Keating, J. , and O'Connell, N., "Random matrix theory and the derivative of the Riemann-zeta function," Proc. R. Soc. London, Ser. A 456, 2611-2627 (2000).

${ }^{13}$ Hughes, C., Keating, J., and O'Connell, N., "On the characteristic polynomials of a random unitary matrix," Commun. Math. Phys. 220, 429-451 (2001).

${ }^{14}$ Keating, J. and Snaith, N., "Random matrix theory and $\zeta(1 / 2+i t)$," Commun. Math. Phys. 214, 57-89 (2000).

${ }^{15}$ Mehta, M., Random Matrices, 2nd ed. (Academic, San Diego, 1991).

${ }^{16}$ Mehta, M. and Normand, J-M., "Moments of the characteristic polynomial in the three ensembles of random matrices," J. Phys. A 34, 4627-4639 (2001).

${ }^{17}$ Strahov, E. and Fyodorov, Y. V., "Universal results for correlations of characteristic polynomials: Riemann-Hilbert approach," math-ph/0210010.

${ }^{18}$ Szegö, G., Orthogonal Polynomials, Vol. 23 of American Mathematical Society, Colloquium Publications, 4th ed. (AMS, Providence, RI, 1975).

${ }^{19}$ Uvarov, V. B., "The connection between systems of polynomials orthogonal with respect to different distribution functions," USSR Comput. Math. Math. Phys. 9, 25-36 (1969). 